\title{
1 Candidate genes under balancing selection in a plant bacterial pathogen
}

José A. Castillo ${ }^{1^{*}} \&$ Spiros N. Agathos ${ }^{1}$

$3 \quad{ }^{1}$ School of Biological Sciences and Engineering, Yachay Tech University, Hacienda San Jose s/n and

4 Proyecto Yachay, Urcuquí, Ecuador.

$5 \quad *$ Corresponding Author

6 jcastillo@yachaytech.edu.ec

7 Keywords: balancing selection, Ralstonia solanacearum, Tajima's D, Watterson's theta, Fu \& Li's D*,

8 virulence related genes, pathogenesis 
11 Plant pathogens are under significant selective pressure by the plant host. Consequently, they are 12 expected to have adapted to this condition or contribute to evading plant defenses. In order to acquire 13 long-term fitness, plant bacterial pathogens are usually forced to maintain advantageous genetic diversity 14 in populations. This strategy ensures that different alleles in the pathogen's gene pool are maintained in a 15 population at frequencies larger than expected under neutral evolution. This selective process, known as 16 balancing selection, is the subject of this work in the context of a common plant bacterial pathogen. We 17 performed a genome-wide scan of Ralstonia solanacearum, an aggressive plant bacterial pathogen that 18 shows broad host range and causes a devastating disease called 'bacterial wilt'. Using a sliding window 19 approach, we analyzed 57 genomes from three phylotypes of $R$. solanacearum to detect signatures of 20 balancing selection. A total of 161 windows showed extreme values in three summary statistics of 21 population genetics: Tajima's D, Watterson's $\theta$ and Fu \& Li's D*. We discarded any confounding effects 22 due to demographic events by means of coalescent simulations of genetic data. The prospective windows 23 correspond to 78 genes that map in any of the two main replicons of $R$. solanacearum. The candidate 24 genes under balancing selection are related to primary metabolism (51.3\%) or directly associated to 25 virulence (48.7\%), being involved in key functions targeted to dismantle plant defenses or to participate in 26 critical stages in the pathogenic process. These genes are useful to understand and monitor the evolution 27 of bacterial pathogen populations and emerge as potential candidates for future treatments to induce 28 specific plant immune responses. 


\section{INTRODUCTION}

30 Balancing selection (BS) is a well-known concept in evolutionary biology and population genetics that has

31 extensively analyzed in many organisms. BS is a type of positive selection that favors the maintenance of

32 a high genetic diversity within a given population. This diversity could be displayed as an excess of

33 polymorphisms on existing alleles or as the maintenance of different alleles at selected loci. Usually BS

34 influences genetic variation in genomes in a localized way, maintaining diversity at the selected sites but

35 also increasing diversity at closely linked neutral sites (7). BS works through different mechanisms, namely,

36 heterozygote advantage (also called overdominant selection) (26), frequency-dependent selection (58)

37 and spatial/temporal heterogeneity (27). One particularly interesting case is frequency-dependence

38 selection that is related to the coevolution between host and pathogen following the 'trench warfare'

39 model. This model postulates that coevolution of both host and pathogen leads to stable richness of

40 polymorphisms through BS (59). Good examples of this model are interactions of plant resistance genes

41 with virulence-related genes of the pathogen under defined ecological and epidemiological conditions

42 specific for each host-pathogen system. In this case, elevated polymorphism levels in pathogen virulence

43 genes have been found in several systems and therefore it is possible to detect them using standardized

44 tests (59).

45 Lately, much attention has been paid to BS in different eukaryotic species such as humans (33; 4), plants

46 (52) and parasites (62), however, very little to bacteria, with one exception, two species of Staphylococcus

47 genus that cause serious diseases in the respiratory tract, skin and other organs of humans $(64 ; 71)$. At the

48 level of plant bacterial pathogens, there are no reported works that indicate whether BS is a significant

49 force that shapes populations, modulates the interaction with the plant host and directs evolution. In this

50 work, we focus on detecting BS events in the far less studied plant host-bacterial pathogen system and

51 take Ralstonia solanacearum as model species to perform the analyses.

$52 R$. solanacearum belongs to the Betaproteobacteria class and the Burkholderiaceae family and is 53 considered a species complex (RSSC) because it is composed of a large number of genetic groups, often 54 subdivisible into a number of clonal lines (22). RSSC has lately been re-classified in three different species 55 (54) based on a previous phylogenetic arrangement that divided the complex into four phylotypes (15). 56 Each phylotype constitutes a major monophyletic cluster of strains and reflects a geographic origin: 57 phylotype I (Asia), phylotype II (Americas), phylotype III (Africa), and phylotype IV (Indonesia)(15; 5). 58 Phylogenetic studies show that phylotype II is also divided into two monophyletic subgroups designated 59 IIA and IIB (5). Strains belonging to this RSSC are aggressive plant pathogens that cause wilt disease of 
more than 250 plant species including economically valuable crops. These bacteria alternate between two lifestyles, as saprophytic on soil and water, and as pathogen inside plant tissues and organs. The bacteria enter susceptible plants through the roots, invade the xylem vessels, form biofilms and spread to the aerial parts of the plants. For pathogenesis, RSSC strains use an ample repertoire of molecular weapons like cell wall degrading enzymes, an extracellular polysaccharide and effectors secreted through the type III secretion system (T3SS) (20). All virulence factors are expressed and eventually secreted in a coordinated manner and appear to have additive effects since no single factor can completely explain infection and disease symptoms (38). At the genomic level, the RSSC strains harbor two DNA circular molecules, a larger replicon of $3.7 \mathrm{Mb}$ and a smaller $2.1 \mathrm{Mb}$ replicon, corresponding to chromosome and megaplasmid respectively. Both replicons contain housekeeping as well as virulence-related genes (55).

To investigate BS in the RSSC, we performed a genome-wide scan on both replicons (chromosome and megaplasmid) and attempted to determine whether BS is more frequent in essential versus virulencerelated genes. Only for the purposes of this work, we have considered each RSSC phylotype (including subgroups IIA and IIB) as single, independent populations and have measured the excess of common polymorphisms using the classical summary statistics (Tajima's D and others) rather than rely on modelbased methods (13) or new summary statistics (like $\beta)(56)$ because it was considered that they would not add more confidence to the results when used together with Tajima's D.

DATA AND METHODS

\section{Sequence data and alignment.}

Fifty-seven full-genome sequences of three RSSC phylotypes were downloaded from NCBI's FTP server (https://www.ncbi.nlm.nih.gov/genome/microbes/) in February and April, 2017. We selected 20 genomes for phylotype I (CQPS-1, FJAT-1458, FJAT-91, FQY_4, GMI1000, KACC10709, OE1-1, PSS1308, PSS190, PSS4, RD13-01, RD15, Rs-09-161, Rs-10-244, Rs-T02, SD54, SEPPX05, T010, UW757, YC45) and phylotype IIB (2310BR, CFBP1416, CFBP3858, CFBP6783, CFBP7014, CIP417, GEO_304, GEO_96, IBSBF1503, IPO1609, Po82, RS 488, RS2, UW163, UW179, UW24, UW365, UW491, UW551, UY031). For phylotypes IIA and IV we used the largest number of genomes available in the database (12 genome sequences: B50, BBAC-C1, CFBP2957, CIP120, Grenada 9-1, IBSBF1900, K60, P597, RS 489, UW181, UW25, UW700; and 5 genome sequences: A2-HR MARDI, KACC 10722, PSI07, R229, R24, respectively). Unfortunately, there was not enough genome sequences for phylotype III at the time we retrieved sequences to perform analyses, therefore we did not include this phylotype in the analysis. All analyses were performed on the main (chromosome) and the secondary (megaplasmid) replicons of the RSSC. 
91 We aligned the genome sequences using progressiveMauve aligner v2.4.0 (12) with default settings. For

92 phylotype IV sequences, we increased the gap penalty (gap open score -600) to avoid opening 93 unnecessarily large gaps, however we allowed small gaps (3-10 bp). For all analyses we used only Locally 94 Collinear Blocks (LCBs) sequences to assure we worked with homologous sites that show maximal 95 collinearity in order to avoid problems of internal genome rearrangements and gene gain and loss. We 96 used stripSubsetLCBs script distributed with Mauve to extract LCBs longer than 1000 bp that were shared 97 by RSSC genomes. This script generates an xmfa file that should be converted to a fasta file to facilitate 98 the ensuing analyzes. For this purpose, we used a Perl script (xmfa2fasta).

\section{Statistical analyses}

100 We applied summary statistics to detect BS. The summary statistics were used to measure an excess of 101 polymorphisms linked to the genomic regions under this type of selection. We adopted three different 102 statistics: Watterson's estimate of theta $\left(\theta_{w}\right)$, Tajima's D, and Fu \& Li's D* $(11 ; 3)$. Tajima's D test takes into 103 account the average pairwise nucleotide diversity between sequences and the number of segregating sites 104 expected under neutrality for a population at mutation-drift equilibrium (60). Tajima's D is useful to detect departures from neutrality when considering an excess of rare alleles indicating positive selection/selective sweep, or the opposite, excess of common alleles that leads to assume BS has operated in the population. In our case, Tajima's D helps to find polymorphisms at intermediate frequency. Watterson's theta measures the population mutation rate which is understood as the product of the effective population size and the neutral mutation rate from the observed nucleotide diversity of a population (69). In this case, $\theta_{w}$ is an indicator of high level of polymorphisms. Fu \& Li's D* statistics considers the number of derived singleton mutations and the total number of derived nucleotide variants without an outgroup (19). We used a combination of these three test statistics to detect excess of common 113 polymorphisms along the allele frequency spectrum relative to expectations under neutral equilibrium.

114 The use of three indicators may seem overly conservative, but it helps to reduce false positives and to 115 detect genes or genome regions that are robust candidates for operating under BS. Neutrality tests were 116 calculated with VariScan 2.0.3 (30) using total number of segregating sites and excluding sites containing 117 gaps or ambiguous nucleotides.

118 We performed a genome-wide scan to find genes or genome regions under BS using a sliding window 119 approach. Thomas and colleagues (ref. 64) tested windows of two sizes, $100 \mathrm{bp}$ and $200 \mathrm{bp}$ for S. aureus 120 genome analysis coming to the conclusion that $200 \mathrm{bp}$ windows is the optimal and $100 \mathrm{bp}$ windows is the 121 second best alternative for a genome scan. The type strain of S. aureus subsp. aureus DSM $20231^{\top}$ has a 
122 genome of 2,9 $\mathrm{Mb}$ (35) which is slightly smaller than the RSSC chromosome (3.7 Mb for reference strain 123 GMI1000) (55). Moreover, the average length of protein-coding genes is similar for both bacterial species 124 [946 bp (chromosome), 1,077 bp (megaplasmid) for RSSC and about 1,009 bp for S. aureus; see ref. 55 and 125 35]. Therefore, a $200 \mathrm{bp}$ windows seems to be an adequate window size for RSSC. All three statistics were 126 calculated for consecutive, non-overlapping, $200 \mathrm{bp}$ windows, and only those windows with the highest $1275 \%$ values coinciding in the three statistics were chosen as possible candidates for further analyses. 128 Windows without single nucleotide polymorphisms (SNPs) among aligned genomes were excluded from 129 analysis because the statistics are calculated based on polymorphisms.

130 Per site mutation $(\theta)$ and recombination $(\rho)$ rates are parameters useful for understanding the recent 131 history of RSSC populations, however they also help to test demographic models to discover which one 132 best fits the observed data for each population (see below). These parameters were estimated using a 133 penalized approximate likelihood coupled to a Bayesian reversible-jump Markov chain Monte Carlo 134 sampling scheme. For this, we set up the starting $\rho$ value to 30 , penalized each block with a value of 10 135 and used the gene conversion model. We run $10^{6}$ chains to obtain $\rho$ and $\theta$ values using the program 136 INTERVAL (41) implemented in the RDP4 package (39). Because RDP was not designed to handle long 137 genomic sequences, we estimated values of $\rho$ and $\theta$ by averaging the obtained values from sets of 50,000 bp each along the length of nucleotide sequence alignments.

139 The summary statistics $\left(\theta_{w}\right.$, Tajima's $D$, and Fu \& Li's $\left.D^{*}\right)$ must be carefully analyzed because different 140 demography scenarios could give similar signals as BS when applied to real population data. For example, 141 different population structures like a contraction or a selective bottleneck could generate confounding 142 indications mimicking BS. To correct potentially confounding effects of demography we need to select 143 adequate null demographic models and test them with real data. For this purpose, we adopted a 144 simulation-based approach to generate genetic statistics under three main demographic scenarios: 145 standard neutral model (SNM), a recent population contraction model (PCM), and a recent bottleneck 146 model (BNM). The SNM assumes a constant-sized population, thus Tajima's D is expected to be zero (60). 147 Under PCM and BNM assumptions, Tajima's D is positive or shows higher values than with SNM, which 148 indicates the abundance of prevalent lineages before a contraction or a bottleneck effect. Simulations 149 were performed under the coalescent simulation framework by employing the algorithm described in 150 Hudson (ref. 29) to infer the coalescent tree with recombination. The PCM assumes that the population 151 has undergone a size reduction at a given time that we fixed at 0.005 coalescent time units before the 152 present, according to Thomas and collaborators (ref. 64). Coalescent time units are measured in $4 N_{e}$ 
153 generations where $N_{e}$ corresponds to the current effective population size (29). For BNM simulations, the

154 model assumes that the population suffered two demographic events, a contraction and then a population

155 growth. In this case, we calibrated time ( $T_{c}$ and $T_{r}$ time of contraction and time of recovery, respectively)

156 for first and second events as 0.005 coalescent time units before the present until a relevant demographic 157 event (64). The reduction of population size $\left(N_{e}\right)$ relative to constant growth was set to 5 , for PCM and for 158 the first and second demographic events of BNM. The fivefold reduction of the original population size is 159 based on the $N_{e}$ decrease reported in experimental studies performed on different bacterial species (47; $16072 ; 10 ; 37)$. Finally, $\rho$ and $\theta$ values calculated previously were used to complete the information required 161 to run the simulations. For each window, we computed 10,000 coalescent simulations using DNASP v. $162 \quad 6.11 .01$ for the three summary statistics under the relevant demographic model (53). A $p$-value was 163 estimated for each window to validate statistically the potential differences between simulated and 164 observed data. Windows with extreme (i.e. significant) $p$-values (at the right tail, $p_{\text {Sim }<O b s}<0.1$ or $p_{\text {Sim< }<0 b s}$ $<0.05)$ for the three statistics and the three demographic models were recorded as highly significant and accepted as candidates under BS. However, windows with significance for only two statistics (Tajima's D

167 and Fu \& Li's D* or Tajima's D and $\theta_{w}$, see Table 2 and Supplementary Table 3) were also accepted as secondarily significant.

\section{Gene identification and function}

170 Sequences of windows with significant values were used to identify genes that overlap in them. For this, 171 Blastn searches were performed using standard settings (2). We used four RSSC reference strains for 172 sequence comparison and gene identifier assignation: GMI1000 for phylotype I; CFBP2957 for phylotype 173 IIA; Po82 for phylotype IIB; and PSI07 for phylotype IV. Uniprot (63) and Pfam (17) databases including 174 their tools were used to retrieve information on the features and function of proteins. The respective gene 175 ontology (GO) term was applied to each identified protein using QuickGO 176 (https://www.ebi.ac.uk/QuickGO/). The KEGG database was used for further understanding putative gene 177 functions, utilities of the bacterial systems and to define orthologs for RSSC genes under BS (31). 178 Identification of T3SS effector proteins was achieved using the web interface named "Ralstonia T3E" 179 (https://iant.toulouse.inra.fr/T3E) with the curated effector repertoire database (45). 
For all analyses performed in this work, we chose to work with locally collinear blocks (LCBs) than with complete genome alignments because LCBs produce aligned and concatenated sequences composed of homologous regions of sequence shared by the genomes under study. In this way, only conserved segments that appear to be internally free from genome rearrangements were considered for population parameters and summary statistics calculations. This is critical for calculations aimed at detecting polymorphisms on aligned sequences. Genome alignments of the RSSC phylotypes analyzed in this work produced a variable number of LCBs that concatenated represent about (or higher than) $50 \%$ of their respective genomes (except for the megaplasmid of phylotype IV, see Table 1). Because some genome sequences from the database are poor in megaplasmid sequences, we were only able to align seven genome sequences for the phylotype IIA megaplasmid (Table 1).

Alignments were analyzed for information on population parameters which are necessary for the simulations (see below). Per site recombination rate $(\rho)$ and per site mutation rate $(\theta)$ vary across different phylogenetic groups in RSSC (Table 1). The chromosome of phylotype IIB and the chromosome of phylotype I show the lowest value for $\rho$ and $\theta$ respectively. On the other hand, the highest values of the two parameters are shared by the megaplasmid of phylotype I (for $\rho$ ) and the megaplasmid of phylotype IIA (for $\theta$ ). Interestingly, the relation $\rho / \theta$ gives opposite values depending on the phylotype. Phylotypes II ( $A$ and $B$ ) and IV show values lower than 1 for $\rho / \theta$, while phylotype I reaches values higher than that. This result suggests that the role played by recombination seems to be uneven across RSSC lineages and that recombination had a stronger influence on introducing nucleotide substitution relative to mutation in phylotype I (both replicons) than in other phylotypes.

\section{Summary statistics}

RSSC genome alignments were scanned for BS signatures in both replicons (i.e. chromosome and megaplasmid). We focused the analysis on phylotype I, II and IV as there were not enough genome sequences available in the databases for phylotype III at the time of the analysis and phylotype II was analyzed in both its subclusters as they were separate and independent phylogenetic groups (Table 1). The extent of polymorphism was measured by using the three summary statistics mentioned above $\left(\theta_{w}\right.$, Tajima's D, and Fu \& Li's D*). The Tajima's D values calculated for the whole replicon of each phylotype ranged from -0,6417465 to 1,084 depending on phylotype (Table 1). Phylotypes I and IV show Tajima's D distribution shifted towards negative values in both replicons, as well as phylotype IIA (megaplasmid). On the contrary, phylotypes IIA (chromosome) and IIB (both replicons) show a tendency towards positive values. Fu \& Li's D* results follow a similar pattern as Tajima's $D$. This suggests that both these statistics 
213 are highly correlated, an aspect that is confirmed later (see below). When we estimated the summary 214 statistics using the sliding window strategy, an ample assortment of values was obtained for each 215 phylotype and replicon. After eliminating windows without SNPs, we observed extreme Tajima's D values

216 (such as 3.46 and -2.506 for the chromosome in phylotype I) but also moderate values, along all windows 217 analyzed (Supplementary Table 1). The tendency towards negative values was reflected in Tajima's D and 218 Fu \& Li's D* mean values of sliding windows analysis for phylotypes I (both replicons), IIA (megaplasmid) 219 and IV (both replicons) (Supplementary Table 1, Figure 1). Watterson's $\theta$ values are relatively high for all 220 phylotypes except for phylotype I and IIB (chromosome). The slight differences between $\theta$ and $\theta_{\mathrm{w}}$ observed 221 in Table 1 are due to the way of calculating this statistic, as in one case, we employed the Bayesian method 222 and in the other the formula proposed by Watterson (ref. 69).

223 A two-dimensional plot of all three statistics suggests that their values are correlated (Figure 1). To confirm 224 a possible correlation between them, we calculated the Spearman rank correlation coefficient between $225 \theta_{w}$, Tajima's D and Fu \& Li's D* using the sliding window data. As expected, results show that there is a 226 strong pairwise correlation among the three statistics for all phylotypes and replicons except for phylotype

227 I when comparing $\theta_{\mathrm{w}}$ and Tajima's D (Supplementary Table 2). In some cases, a very high positive 228 correlation was observed, as is the case of phylotypes IIA and IV for Tajima's D-Fu \& Li's D* combination $229(0.738,0.964$ and $0.982,0.976$, respectively) suggesting a strong agreement between these statistics. This 230 result also supports the idea that the high values of the statistics point out to real BS signatures (or 231 demographic structuring) in aligned sequences rather than being random values.

\section{Simulations and candidate genes under balancing selection}

233 We tested whether the unusual incidence of high values of summary statistics obtained from aligned 234 sequences was due to BS on RSSC genomes or reflected effects of demography. We adopted the widely 235 used strategy based on simulation of genetic data under the coalescent framework. Three most plausible 236 demographic scenarios were tested (SNM, PCM and BNM) as null models. Although these models may not 237 represent the exact history of RSSC populations because of their intrinsic complexity, this approximation 238 is sufficiently advantageous to be used as a null demographic model focused upon reducing false positives. 239 We included in our analysis the gene cluster agr from S. aureus as a positive control (64). We analyzed $2403,537 \mathrm{bp}$ of the agr cluster using the standard procedure for BS signature detection in RSSC aligned 241 sequences as detailed in the Data and Methods section. This analysis produced 18 windows, however, in 242 none of them, we obtained maximum matching values for the three statistics. As expected, windows with 243 high observed values of Tajima's $D, \theta_{w}$, or Fu \& Li's D* showed very significant values after simulations 
244 according to the demographic models tested in this work (observed values: Tajima's $D=2.72677^{* *} ; \theta_{\mathrm{w}}=$ $2450.05249 * * ;$ Fu \& Li's $D^{*}=1.73125^{* *}$, the double asterisk meaning significant difference at $p<0.05$ 246 compared to values obtained with simulations for SNM). After having demonstrated confidence in the 247 analysis using this positive control, we applied the same procedure to scan the RSSC aligned sequences. 248 Results show (Table 1 ) that the top 5\% of the distribution of the summary statistics exceed the respective 249 simulated values (under the corresponding demographic model) in most of the cases, as validated by 250 hypothesis testing significance. Note that the power of this detection resides in the concurrent 251 consideration of all three statistics, Tajima's D, $\theta_{w}$, and Fu \& Li's D*. This result provides a robust evidence 252 that the windows with high values of summary statistics correspond to genes or genomic regions under 253 BS (Table 1). Subsequently, we identified the genes overlapping the candidate windows (Table 2). A list of 254 unidentified genes (unknown gene function) or windows corresponding to intergenic regions is detailed in 255 Supplementary Table 3.

256 In general terms, the results show that BS affects more frequently coding regions than non-coding 257 sequences in RSSC genomes (compare Table 2 with Supplementary Table 3). We found 161 windows with 258 significant values for the three statistics. Demography simulations reduced the number to 142 significant 259 windows that correspond to 78 known genes (Table 1) and 22 intergenic regions or genes with unknown 260 identity or function (Supplementary Table 3). This may indicate that 19 windows are probably false 261 positives. The percentage of genes detected under BS is low $(1.7 \%, 78$ genes out of 4,585 which is the 262 median protein-coding genes in RSSC according to the Genome Database, 263 https://www.ncbi.nlm.nih.gov/genome/?term=Ralstonia+solanacearum). This result is consistent with 264 other analyses in eukaryotic systems like humans (4) or plants (52) and also in prokaryotes (64) that stress 265 the rarity of finding BS signatures on sequence genomes. The candidate genes under BS are described 266 below according to phylotype and replicon.

267 Phylotype I. We detected 440 windows for the chromosome of this phylogenetic group at the top $5 \%$ of 268 the distribution, however only 21 showed concurrent high values in all three summary statistics and 14 269 were recorded as highly significant after the simulation process.

270 We found seven peak values of Tajima's D, $\theta_{\mathrm{w}}$ and Fu \& Li's D statistics on phcB (five extreme values) and 271 phcS (two extreme values, Figure 2, Table 2) genes. These two genes are arranged in an operon together 272 with a third gene named $p h c R$. The gene $p h c B$ encodes a SAM-dependent methyltransferase that 273 synthesizes 3-hydroxy palmitic acid methyl ester (3-OH PAME), a quorum-sensing signal that accumulates 274 in the extracellular space when the bacteria are multiplying rapidly in a restricted space (18). Quorum- 
sensing is a key process regulating and synchronizing the expression of specific genes involved in biofilm formation, pathogenicity, and production of secondary metabolites like siderophores, exoproteases, and exotoxins (36). PhcS (histidine kinase) and phcR (response regulator) genes code for elements of a twocomponent regulatory system that responds to threshold concentrations of 3-OH PAME by elevating the level of functional PhcA, the fourth component of the system (8). PhcA is the global virulence regulator in RSSC since it regulates hundreds of genes directly involved in pathogenesis but also in basal metabolism and cell homeostasis $(34 ; 48)$.

Another gene showing multiple peaks in statistics values is RSc2066 that codes for a haloacid dehalogenase-like hydrolase (Table 2). In this case, four consecutive high values of the statistics suggest that this gene is likely under BS. This enzyme has a hydrolase activity that cleaves different bonds (i.e. C$\mathrm{O}, \mathrm{C}-\mathrm{N}, \mathrm{C}-\mathrm{C})$, however its exact role at the cellular level is unknown.

In this phylotype we also found genes related to basic metabolism like a glycosyl transferase and an operon consisting of two genes, IrgAB, that modulates murein hydrolase activity which is linked to biofilm dispersal and cell lysis (24). These IrgAB genes intervene indirectly in pathogenesis since an essential step in this process is the formation and dispersal of biofilms in RSSC (36).

For the megaplasmid we found 304 out of 6162 windows with highest Tajimas's $D, \theta_{w}$, and Fu \& Li's D* values. After simulation for relevant demographic models, only nine windows generated significant values.

Some interesting genes associated to virulence were observed in this replicon (Table 2). We identified three different T3SS effector genes as targets for BS: ripD of the avrPphD family; ripA4 and ripU. 294 Interestingly, both ripD and ripU show two significant hits (two windows with significant values) along their 295 coding sequences. RipU is part of the core-effectome within the RSSC as well as ripA4 that is common in effector collections and plays an important role in the interaction between $R$. solanacearum and the pepper plant (46). Another gene, uxuL (RSp0832) codes for the main glucuronolactone/galactarolactone lactonase in the genome of the GMI1000 strain. UxuL is organized within an operon with three other genes: garD encodes a D-galactarate dehydratase, RSc0831 a putative NAD-dependent epimerase/dehydratase and pehC a polygalacturonase. PehC is an enzyme related to virulence since it cleaves oligomers of galacturonate, however its exact role is unknown. It was hypothesized that PehC acts by degrading plant oligogalacturonate signal molecules that elicit production of reactive oxygen species

303 (ROS) as a defense response. This degradation would reduce tomato antimicrobial responses and increase bacterial virulence (23). This operon is regulated by GulR, a transcription factor of the LysR family involved 
305

in glucuronate utilization and metabolism. Downstream of this operon is located exuT, the galacturonate transporter gene.

Conversely, genes that are not directly related to virulence but to primary metabolism were also identified in megaplasmid aligned sequences: A probable pullulanase related glycosidase protein (PulA) that might work like a glycogen debranching enzyme; a polyphenol oxidase (laccase) oxidoreductase and a putative signal sensing transmembrane protein with phosphorelay sensor kinase activity. Lastly, a significant window matched with an intergenic region surrounded by a hybrid sensor histidine kinase/response regulator and upstream of an integrase related to phage or transposon insertion (Supplementary Table 3).

Phylotype IIA. At the chromosome level we selected 444 windows that showed $5 \%$ highest scores in each statistic. From these, 21 windows showed highest values for all three statistics and also significant values on simulations with respective demographic models.

The first genes that appear in the list are those involved in essential cell functions. There are various enzymatic functions (i.e. a 3-hydroxybutyryl-coA dehydrogenase oxidoreductase, an isoleucine-tRNA ligase, a transcription regulator and others, Table 2) and diverse transporters (a permease from the liv operon, a binding-protein-dependent transporter). Among this group, a gene that attracted our attention is adi which encodes a lysine decarboxylase (LDC). This gene and other related genes (arginine and ornithine decarboxylases) are directly involved in amino acids metabolism but indirectly in pathogenesis. Studies on other bacterial species indicate that these genes are implicated in stress response against the low $\mathrm{pH}$ in the medium $(44 ; 43)$ and against oxidative stress and chemical quenching induced by the host (66). This gene product or LDC metabolic products also intervene in cell adhesion to host tissues (67)

Among the genes related to virulence and survival, we found two contiguous genes, $p h c Q$ and another one downstream from it showing elevated values of selection statistics. PhcQ is a response regulator receiver, from the CheY family and part of the $p h c B S R Q$ operon that regulates PhcA, the master regulator that positively and negatively regulates many genes responsible for pathogenicity in RSSC (70). The gene contiguous to $p h c Q$ encodes a methyltransferase, however it is not known if $P$ hcQ participates in quorum sensing as does the main methyltransferase, PhcB. Two additional genes were associated to BS signatures: srkA and RCFBP_21242. The srkA gene encodes a stress response kinase A, which probably counteracts the accumulation of ROSs produced by the host and protects the bacterial cell from antimicrobial and environmental stressors in a similar way to the YihE protein kinase of Escherichia coli (14). RCFBP_21242 encodes a putative isomerase with a phenazine biosynthesis (PhzC/PhzF) domain. Phenazines constitute a large group of nitrogen-containing heterocyclic compounds produced by bacteria and show an ability to 
handle ROS, contribute to biofilm formation, cell adhesion and enhance bacterial survival, among other activities (50).

338 Results on the T3SS effector repertoire analysis of phylotype IIA-chromosome showed a number of genes 339 with a BS signature: ripM, ripW, ripG4 (formerly GALA4) and ripS5 (formerly SKWP5). RipG4 and ripW were 340 associated to two significant windows each suggesting these genes are clearly under BS. Since we have 341 used the CFBP2957 strain as reference for gene identification, we find that this strain has an insertion of a 342 transposon encoding a transposase (RCFBP_20595) in the ripS5 gene, therefore this appears to be a 343 pseudogene copy of this effector. Most of the phylotype IIA strains show a disruption in the ripS5 gene 344 due to transposon insertions, however there are some strains harboring the complete gene (i.e. the 345 RS_489 strain)(45).

346 At the megaplasmid level, phylotype IIA showed six genes with significant signatures of BS after filtering 347 with coalescent simulations: one related to basic metabolism (cyaB, an $A B C$ transporter) and four 348 pertaining to pathogenicity: a putative adhesin/hemolysin that plays a significant role in cell adhesion; a 349 cardiolipin synthase A, from the phospholipase D family, involved in membrane biosynthesis and toxin 350 production and resistance (61); a putative Type IV fimbrial component, encoded by the pilY1 gene 351 participating in Type IV pili biosynthesis (type IV pili are essential for adhesion and pathogenesis)(1), and 352 a bacteriophage-related protein with unknown function. Finally, a T3SS effector named ripF1 [formerly 353 PopF1] that is very well characterized (42).

354 Phylotype IIB. Three hundred fifty two windows corresponding to the top $5 \%$ of the distribution were 355 analyzed for the chromosome. Only 33 windows showed highest values of the three statistics concurrently, 356 but 23 windows showed significant values after coalescent simulations.

357 The most abundant group of genes identified in this chromosome are those implicated in primary 358 metabolism with an ample diversity that varies from genes encoding metabolic enzymes (synthases, 359 epimerases, etc.) to a number of permeases and other transporter related genes (Table 2). Again, an amino 360 acid decarboxylase was found within this group.

361 Various genes are linked to virulence: a key component of pili biogenesis (Type IV pili assembly protein $362 \mathrm{PilX}$ ) and the gene responsible for the production of the molecule that mediates quorum sensing, $p h c B$ 363 were identified. Among genes encoding T3SS effectors, three were most notable (ripAJ, ripG6 and ripG7) 364 and multiple windows enriched two of them (two and three hits for ripAJ and ripG7, respectively, Table 2). 
365

366

367

368

369

370

371

372

373

374

375

376

377

378

379

380

381

382

383

384

385

386

Interestingly, a conserved protein (RSPO_c02827) showed also two significant hits along its sequence but its function is unknown (Supplementary Table 3).

We identified 26 windows with significant values distributed across the megaplasmid after the simulation process. Since many virulence-related genes reside in the megaplasmid, it was not surprising to have identified many of them. Ten different T3SS effector genes were found (Table 2) and some were noted by redundant windows as is the case of genes ripH2 (9 hits), ripS3 (3 hits), ripBH (3 hits), ripAR (2 hits) and ripF1 ( 2 hits). On the other hand, only few genes involved in basic metabolism were identified: an enoyl reductase (NADH dependent) and two contiguous genes, polygalacturonase and gluconolactonase, that overlap within a single window ( $\mathrm{N}$-terminus of the first and $\mathrm{C}$-terminus of the second enzyme).

Phylotype 4. The chromosome showed 463 windows in the top $5 \%$ of the distribution for each statistic, and after selection for the matching values in the three statistics and the simulation, only 15 were retained as highly significant for further analyses.

We found interesting genes in the chromosome such as one encoding the RNA polymerase sigma 70 factor which gathered three consecutive windows. Other genes that received multiple hits include a tyrosyl-tRNA synthetase, a glucose-1-phosphate uridylyltransferase and a putative ABC-type transporter. On the other hand, a phospho- $\mathrm{N}$-acetylmuramoyl-pentapeptide transferase was detected by one window. In the gene group related to virulence, we found two T3SS effector genes with multiple windows: ripE1 from the AvrPphE family and ripW [formerly PopW], a hairpin with a pectate lyase domain.

At the megaplasmid level, we found only two metabolically essential genes with significant values: a putative acetyltransferase and a chloride channel clcB-like protein.

\section{DISCUSSION}

In this work, we report the systematic exploration of the genomes belonging to the main RSSC phylotypes with the intention of finding signatures of BS. To our knowledge this is the first time that a bacterial plant pathogen is analyzed for this type of selection at the genomic level. The analysis was performed on the main replicons of RSSC (chromosome and megaplasmid), but not on small plasmids, phages or mobile genetic elements. We scanned genome sequences using a sliding window approach and subsequently applied widely used summary statistical tests aimed at detecting the excess of polymorphisms on $200 \mathrm{bp}$ window sequences: Watterson estimator theta, Tajima's D, and Fu \& Li's D*. We chose to use these tests rather than other strategies (i.e. model based methods) because of their simplicity, wide range of BS forms detected and broad access to diverse software tools. This strategy together with exhaustive coalescent 
simulations to correct confounding effects of demography was an effective approach to reach our objective to detect genes and genomic regions under BS in RSSC. Tajima's D is useful for detecting intermediate and ancient signatures of BS. In contrast Fu \& Li's D* and $\theta_{w}$ help to identify relatively recent instances of this type of selection. Our approach may be overly conservative, and hence we might have missed some genuine occurrences of BS. However, it may have conferred more certainty to the positive hits found on RSCC genomes. Indeed, we detected dozens of gene candidates in RSSC genomes in agreement with Fijarczyk and Babik (ref. 16) who recognized this is common in pathogens' genomes.

402 The results confirm the validity of the methodological strategy used and add new insights to understand 403 RSSC and plant host interaction. We have found many bacterial genes that show unambiguously features 404 of being under BS. The phcBRS operon scored 7 significant windows in phylotype I and one in phylotype 405 IIA and one in phylotype IIB, indicating this genomic region is under strong BS. Remarkably, Guidot and 406 collaborators (ref. 25) also found that one component of this system, phcS, was subject to strong selection 407 from the plant host given the evidence that this gene was targeted by mutations in an in planta 408 experimental evolution system. The phcBRS is responsible for controlling a complex regulatory network 409 that responds to environmental conditions and bacterial cell density. This system comprises a two410 component signaling system composed of a histidine kinase, PhcS, that phosphorylates the response 411 regulator, PhcR, when the signal molecule has reached the threshold level and PhcB is the enzyme 412 responsible for forming the signaling molecule that mediates a quorum sensing communication. The main 413 quorum sensing signaling molecules are 3-OH PAME (methyl 3-hydroxypalmitate) or 3-OH MAME (methyl 414 3-hydroxymyristate) depending on the producer strain (28). The key player in this network is a global 415 transcriptional regulator, PhcA, that coordinates the expression of several virulence-related genes 416 including those responsible for the major extracellular polysaccharide, cell wall degrading enzymes, T3SS 417 effectors, and others representing a total of 383 genes (48). Interestingly, an equivalent but simpler 418 network in S. aureus, the agr locus, is also a two-component signal transduction system (membrane-bound 419 histidine kinase sensor, AgrC and transcriptional regulator, AgrA), with a signal molecule (an auto-inducing 420 peptide, $A g r D)$ and a protein responsible for the maturation and export of the signal molecule (AgrB). 421 Again, the key component in this system is the master transcriptional regulator AgrA that binds onto the 422 promoter region and induces transcription from two divergent promoters, P1 and P2 (65). Although this 423 system does not show homology at the sequence level with the phcBRS system in RSSC, it is functionally 424 analogous since it leads to up and down-regulation of over 70 genes, 23 of which are known to be directly 425 related to virulence (21). Interestingly, the agr locus has the strongest known signatures of BS in bacteria 
426

427

428

429

430

431

432

433

434

435

436

437

438

439

440

441

442

443

444

445

446

447

448

449

450

451

452

453

454

455

456

457

to date due to the high number of common polymorphisms. For this reason, the agr locus has been proposed as the positive control of BS for further studies in bacteria (64).

We have also found a set of genes with strong BS signatures whose function is related to adhesion, motility and biofilm formation. Genes encoding Type IV fimbrial biogenesis proteins were found in phylotype IIA (megaplasmid, pilY1) and phylotype IIB (chromosome, pilX). These proteins are essential for the assembly and function of Type IV pili, filamentous structures that mediate bacterial adhesion to surfaces including host cells. This adhesion is tightly linked to the bacterial pathogens' ability to promote the formation of microcolonies and biofilms as well as to their twitching motility and virulence $(32 ; 57)$. In phylotype I (chromosome), we found two LytSR-regulated genes, $\operatorname{Irg} A$ and $\operatorname{Irg} B$ that code for a murein hydrolase exporter and a protein having murein hydrolase activity respectively (24). Both proteins are required for biofilm dispersal that is accompanied by cell lysis and death. Biofilm formation and disruption is a critical step in the process of infection and pathogenesis for RSSC strains. Diverse types of molecules mediate the release of the cells from biofilms, including degrading enzymes (among them, murein hydrolases), nucleases and others $(40 ; 68)$. Additionally, we identified one gene under BS that seems to be directly related to the biosynthesis of phenazines in phylotype IIA. Phenazines constitute a large group of nitrogencontaining heterocyclic compounds produced by a wide range of bacteria, with diverse physiological functions. Among these, they influence swarming motility and biofilm architecture through a not fully understood mechanism (51).

T3SS effectors are key virulence factors at the forefront of the arsenal that RSSC strains harbor to infect plants and achieve full pathogenicity including the metabolic adaptation to parasitic life in the plant (9). T3SS effectors are delivered to plant cells through a proteinaceous needle-like structure, and once inside, they manipulate plant cell metabolism to suppress or evade defense responses and promote bacterial multiplication (6). R. solanacearum strains possess a large repertoire, with 94 effectors identified among RSSC sequenced (45). We found an ample collection of T3SS effector genes with moderate to very strong BS signatures in all phylotypes studied here (Table 2). Some of them belong to very well-known families of effectors like the GALA (ripG4, in phylotype IIA, chromosome; ripG6 and ripG7 in phylotype IIB, chromosome; ripG3, in phylotype IIB, megaplasmid), SKWP (ripS5 in phylotype IIA, chromosome; ripS3, in phylotype IIB, megaplasmid), HLK (ripH2 in phylotype IIB, megaplasmid) and PopF type III translocators (ripF1). Interestingly, some effectors overlap with more than two windows and in different phylotypes (ripW, in phylotype IIA, chromosome and phylotype IV, chromosome), or in the same phylotype (ripD, 
phylotype I, megaplasmid ripU, phylotype I, megaplasmid, ripW and ripG4 in phylotype IIA, chromosome;

ripAJ and ripG7 phylotype IIB, chromosome; ripH2, ripS3, ripAR, ripBH and ripF1 in phylotype IIB, megaplasmid; ripE1_1 and ripW in phylotype IV, chromosome), providing strong evidence that these genes are under BS.

Although genes dedicated to tasks of basal metabolism may seem less relevant for pathogenesis, they also play an important role in the interaction with the plant host and virulence. Peyraud and collaborators (ref. 49) developed a model system to study robustness and metabolic responses to internal and environmental perturbations in $R$. solanacearum. One of their findings highlight the active participation of primary metabolism in sustaining virulence, by activating functionally redundant reactions which may require redundant alleles to satisfy cellular demands including virulence. The expression of virulence factors (such as the exopolysaccharide) is controlled by the virulence regulatory network (VRN) that operate overlapping genes or operons involved in amino acid synthesis (49). While we did not particularly seek redundant or duplicate alleles in this work, we found a number of genes of primary metabolism that perform similar functions at the cellular level. For example, in the set of genes showing BS signatures there are two glucuronolactonases (carbohydrate metabolism), two aminoacyl-tRNA synthetases and two aminoacyl-decarboxylases (amino acid metabolism). These genes have roles in primary metabolism and probably are indirectly playing an essential role in virulence. Another group of genes that we should not neglect are those involved on defense and reduction of toxicity by metabolites produced by the plant host defense mechanisms. In the list of candidate genes under BS we can count a stress response kinase A (srkA) and a number of membrane transporters ( $A B C$ transporters and other permeases, see Table 2). Genes participating on defense pathways were also enriched in S. aureus genome analysis for BS signatures (64).

Analyses of BS operating on bacterial pathogens are particularly relevant to understand the dynamics of plant-microbe interactions. Host-pathogen coevolution leads to maintenance of high variation in genetic selected sites and nearby sequences. In plants, some loci involved in defense processes have highly polymorphic sequences that favor the occurrence of different resistance alleles (7). In pathogens, there is an equivalent scenario in which the pathogen maintains a high variation of polymorphisms in order to take advantage of the plant; consequently, the host-pathogen coevolution directs towards stable balanced polymorphisms and a high number of alleles in both host and pathogen populations (known as the trench warfare model)(59). Interestingly, in RSSC genomes, we found high variation in T3SS effector genes and 
490

491

492

493

494

495

496

497

498

499

500

501

502

503

504

505

506

507

508

509

510

511

512

which may be under significant selection pressure by the plant host. Considering that RSSC has the ability to infect a large number of different plant species (20), it is not rare to find this high variation in the virulence factors. Some effectors (the so-called avirulence proteins) are recognized by proteins encoded by the plant $\mathrm{R}$ genes, however escape from host recognition is possible through fixing mutations on genes coding for effectors or other virulence proteins that increase variation. In order to evade plant detection and defense response, RSSC may tend to favor the maintenance of various allele alternatives (observed in the form of BS), which at the same time increases pathogen fitness. In a more applied sense, the identification of genes under BS, as illustrated in this work, opens the possibility to develop strategies towards establishing long term resistance or tolerance to pathogens in plants. These genes are potential targets for plant immunity, hence potential candidates to engineer broad disease resistance in agriculturally relevant plants.

\section{Acknowledgement}

We thank Mrs. Helen M. Guigues for her valuable assistance with tables and figures.

\section{Funding Statement}

The authors received no specific funding for this work.

\section{References}

1. Alm, R.A., Mattick, J.S., 1997. Genes involved in the biogenesis and function of type-4 fimbriae in Pseudomonas aeruginosa. Gene 192, 89-98. https://doi.org/10.1016/S0378-1119(96)00805-0

2. Altschul, S.F., Gish, W., Miller, W., Myers, E.W., Lipman, D.J., 1990. Basic Local Alignment Search Tool. Journal of Molecular Biology 215, 403-410. https://doi.org/10.1016/S0022-2836(05)80360-2

3. Amambua-Ngwa, A., Tetteh, K.K.A., Manske, M., Gomez-Escobar, N., Stewart, L.B., Deerhake, M.E., Cheeseman, I.H., Newbold, C.I., Holder, A.A., Knuepfer, E., Janha, O., Jallow, M., Campino, S., Maclnnis, B., Kwiatkowski, D.P., Conway, D.J., 2012. Population genomic scan for candidate signatures of balancing selection to guide antigen characterization in malaria parasites. PLoS Genetics 8 , e1002992. https://doi.org/10.1371/journal.pgen.1002992 
4. Andrés, A.M., Hubisz, M.J., Indap, A., Torgerson, D.G., Degenhardt, J.D., Boyko, A.R., Gutenkunst, R.N., White, T.J., Green, E.D., Bustamante, C.D., Clark, A.G., Nielsen, R., 2009. Targets of balancing selection in the human genome. Molecular Biology and Evolution 26, 2755-2764.

$$
\text { https://doi.org/10.1093/molbev/msp190 }
$$

5. Castillo, J.A., Greenberg, J.T., 2007. Evolutionary dynamics of Ralstonia solanacearum. Applied and Environmental Microbiology 73, 1225-1238. https://doi.org/10.1128/AEM.01253-06

6. Chang, J.H., Desveaux, D., Creason, A.L., 2014. The ABCs and 123s of bacterial secretion systems in plant pathogenesis. Annual Review of Phytopathology 52, 317-345. https://doi.org/10.1146/annurevphyto-011014-015624

7. Charlesworth, D., 2006. Balancing selection and its effects on sequences in nearby genome regions.

$$
\text { PLoS Genetics 2, e64. https://doi.org/10.1371/journal.pgen.0020064 }
$$

8. Clough, S.J., Lee, K.E., Schell, M.A., Denny, T.P., 1997. A two-component system in Ralstonia (Pseudomonas) solanacearum modulates production of PhcA-regulated virulence factors in response to 3-hydroxypalmitic acid methyl ester. Journal of Bacteriology 179, 3639-3648. https://doi.org/10.1128/jb.179.11.3639-3648.1997

9. Coll, N.S., Valls, M., 2013. Current knowledge on the Ralstonia solanacearum type III secretion system: The $R$. solanacearum type III secretion system. Microbial Biotechnology 6, 614-620. https://doi.org/10.1111/1751-7915.12056

10.Croucher, N.J., Hanage, W.P., Harris, S.R., McGee, L., van der Linden, M., de Lencastre, H., Sá-Leão, R., Song, J.-H., Ko, K., Beall, B., Klugman, K.P., Parkhill, J., Tomasz, A., Kristinsson, K.G., Bentley, S.D., 2014. Variable recombination dynamics during the emergence, transmission and 'disarming' of a multidrug-resistant pneumococcal clone. BMC Biology 12, 49. https://doi.org/10.1186/1741-7007-1249

11.Croze, M., Wollstein, A., Božičević, V., Živković, D., Stephan, W., Hutter, S., 2017. A genome-wide scan for genes under balancing selection in Drosophila melanogaster. BMC Evolutionary Biology 17. https://doi.org/10.1186/s12862-016-0857-z

12.Darling, A.E., Mau, B., Perna, N.T., 2010. ProgressiveMauve: Multiple genome alignment with gene gain, loss and rearrangement. PLoS ONE 5, e11147. https://doi.org/10.1371/journal.pone.0011147

13.DeGiorgio, M., Lohmueller, K.E., Nielsen, R., 2014. A model-based approach for identifying signatures of ancient balancing selection in genetic data. PLoS Genetics 10, e1004561. https://doi.org/10.1371/journal.pgen.1004561 
14.Dorsey-Oresto, A., Lu, T., Mosel, M., Wang, X., Salz, T., Drlica, K., Zhao, X., 2013. YihE kinase Is a central regulator of programmed cell death in bacteria. Cell Reports 3, 528-537. https://doi.org/10.1016/j.celrep.2013.01.026

15.Fegan, M., and P. Prior. 2005. How complex is the "Ralstonia solanacearum species complex," p. 449462. In C. Allen, P. Prior, and A. C. Hayward (ed.), Bacterial wilt disease and the Ralstonia solanacearum species complex. APS Press, Madison, WI.

16.Fijarczyk, A., Babik, W., 2015. Detecting balancing selection in genomes: limits and prospects. Molecular Ecology 24, 3529-3545. https://doi.org/10.1111/mec.13226

17.Finn, R.D., Coggill, P., Eberhardt, R.Y., Eddy, S.R., Mistry, J., Mitchell, A.L., Potter, S.C., Punta, M., Qureshi, M., Sangrador-Vegas, A., Salazar, G.A., Tate, J., Bateman, A., 2016. The Pfam protein families database: towards a more sustainable future. Nucleic Acids Research 44, D279-D285. https://doi.org/10.1093/nar/gkv1344

18.Flavier, A.B., Clough, S.J., Schell, M.A., Denny, T.P., 1997. Identification of 3-hydroxypalmitic acid methyl ester as a novel autoregulator controlling virulence in Ralstonia solanacearum. Molecular Microbiology 26, 251-259. https://doi.org/10.1046/j.1365-2958.1997.5661945.x

19. Fu, Y.X., Li, W.H., 1993. Statistical Tests of Neutrality of Mutations. Genetics 133, 693-709.

20.Genin, S., Denny, T.P., 2012. Pathogenomics of the Ralstonia solanacearum Species Complex. Annual Review of Phytopathology 50, 67-89. https://doi.org/10.1146/annurev-phyto-081211-173000

21.George, E.A., Muir, T.W., 2007. Molecular mechanisms of agr quorum sensing in virulent Staphylococci. ChemBioChem 8, 847-855. https://doi.org/10.1002/cbic.200700023

22.Gillings, M.R., Fahy, P., 1994. Genomic Fingerprinting: towards a unified view of the Pseudomonas solanacearum species complex. In Bacterial wilt: the disease and its causative agent, Pseudomonas solanacearum, edited by A. C. Hayward and G. L. Hartman. Wallingford: CAB International

23.González, E.T., Allen, C., 2003. Characterization of a Ralstonia solanacearum operon required for polygalacturonate degradation and uptake of galacturonic acid. Molecular Plant-Microbe Interactions 16, 536-544. https://doi.org/10.1094/MPMI.2003.16.6.536

24.Groicher, K.H., Firek, B.A., Fujimoto, D.F., Bayles, K.W., 2000. The Staphylococcus aureus IrgAB operon modulates murein hydrolase activity and penicillin tolerance. Journal of Bacteriology 182, 1794-1801. https://doi.org/10.1128/JB.182.7.1794-1801.2000

25.Guidot, A., Jiang, W., Ferdy, J.-B., Thébaud, C., Barberis, P., Gouzy, J., Genin, S., 2014. Multihost experimental evolution of the pathogen Ralstonia solanacearum unveils genes involved inadaptation to plants. Molecular Biology and Evolution 31, 2913-2928. https://doi.org/10.1093/molbev/msu229 
584

26. Hedrick, P.W., 2012. What is the evidence for heterozygote advantage selection? Trends in Ecology \& Evolution 27, 698-704. https://doi.org/10.1016/j.tree.2012.08.012

27.Herdegen, M., Babik, W., Radwan, J., 2014. Selective pressures on MHC class II genes in the guppy (Poecilia reticulata) as inferred by hierarchical analysis of population structure. Journal of Evolutionary Biology 27, 2347-2359. https://doi.org/10.1111/jeb.12476

28. Hikichi, Y., Mori, Y., Ishikawa, S., Hayashi, K., Ohnishi, K., Kiba, A., Kai, K., 2017. Regulation involved in colonization of intercellular spaces of host plants in Ralstonia solanacearum. Frontiers in Plant Science 8. https://doi.org/10.3389/fpls.2017.00967

29.Hudson, R.R., 2002. Generating samples under a Wright-Fisher neutral model of genetic variation. Bioinformatics 18, 337-338. https://doi.org/10.1093/bioinformatics/18.2.337

30. Hutter, S., Vilella, A.J., Rozas, J., 2006. Genome-wide DNA polymorphism analyses using VariScan. BMC Bioinformatics 10. https://doi.org/10.1186/1471-2105-7-409

31.Kanehisa, M., Furumichi, M., Tanabe, M., Sato, Y., Morishima, K., 2017. KEGG: new perspectives on genomes, pathways, diseases and drugs. Nucleic Acids Research 45, D353-D361. https://doi.org/10.1093/nar/gkw1092

32.Kang, Y., Liu, H., Genin, S., Schell, M.A., Denny, T.P., 2002. Ralstonia solanacearum requires type 4 pili to adhere to multiple surfaces and for natural transformation and virulence: $R$. solanacearum type 4 pili. Molecular Microbiology 46, 427-437. https://doi.org/10.1046/j.1365-2958.2002.03187.x

33.Key, F.M., Teixeira, J.C., de Filippo, C., Andrés, A.M., 2014. Advantageous diversity maintained by balancing selection in humans. Current Opinion in Genetics \& Development 29, 45-51. https://doi.org/10.1016/j.gde.2014.08.001.

34.Khokhani, D., Lowe-Power, T.M., Tran, T.M., Allen, C., 2017. A single regulator mediates strategic switching between attachment/spread and growth/virulence in the plant pathogen Ralstonia solanacearum. mBio 8, e00895-17. https://doi.org/10.1128/mBio.00895-17

35.Kim, B.-S., Yi, H., Chun, J., Cha, C.-J., 2014. Genome sequence of type strain of Staphylococcus aureus subsp. aureus. Gut Pathogens 6, 6. https://doi.org/10.1186/1757-4749-6-6

36.Kumar, J.S., Umesha, S., Prasad, K.S., Niranjana, P., 2016. Detection of quorum sensing molecules and biofilm formation in Ralstonia solanacearum. Current Microbiology. https://doi.org/10.1007/s00284015-0953-0

37.Lapierre, M., Blin, C., Lambert, A., Achaz, G., Rocha, E.P.C., 2016. The impact of selection, gene conversion, and biased sampling on the assessment of microbial demography. Molecular Biology and Evolution 33, 1711-1725. https://doi.org/10.1093/molbev/msw048 
38.Lonjon, F., Turner, M., Henry, C., Rengel, D., Lohou, D., van de Kerkhove, Q., Cazalé, A.-C., Peeters, N., Genin, S., Vailleau, F., 2016. Comparative secretome analysis of Ralstonia solanacearum Type 3 secretion-associated mutants reveals a fine control of effector delivery, essential for bacterial pathogenicity. Molecular \& Cellular Proteomics 15, 598-613.

https://doi.org/10.1074/mcp.M115.051078

39. Martin, D.P., Murrell, B., Golden, M., Khoosal, A., Muhire, B., 2015. RDP4: Detection and analysis of recombination patterns in virus genomes. Virus Evolution 1. https://doi.org/10.1093/ve/vev003

40. McDougald, D., Rice, S.A., Barraud, N., Steinberg, P.D., Kjelleberg, S., 2012. Should we stay or should we go: mechanisms and ecological consequences for biofilm dispersal. Nature Reviews Microbiology 10, 39-50. https://doi.org/10.1038/nrmicro2695

41. McVean, G.A., Myers, S.R., Hunt, S., Deloukas, P., Bentley, D.R., Donnelly, P. 2004. The Fine-Scale Structure of Recombination Rate Variation in the Human Genome. Science 304, 581-584. https://doi.org/10.1126/science.1092500

42.Meyer, D., Cunnac, S., Gueneron, M., Declercq, C., Van Gijsegem, F., Lauber, E., Boucher, C., Arlat, M., 2006. PopF1 and PopF2, Two proteins secreted by the Type III protein secretion system of Ralstonia solanacearum, are translocators belonging to the HrpF/NopX family. Journal of Bacteriology 188, 4903-4917. https://doi.org/10.1128/JB.00180-06

43.Moreau, P.L., 2007. The lysine decarboxylase CadA protects Escherichia coli starved of phosphate against fermentation acids. Journal of Bacteriology 189, 2249-2261.

$$
\text { https://doi.org/10.1128/JB.01306-06 }
$$

44.Park, Y.-K., Bearson, B., Bang, S.H., Bang, I.S., Foster, J.W., 1996. Internal pH crisis, lysine decarboxylase and the acid tolerance response of Salmonella typhimurium. Molecular Microbiology 20, 605-611. https://doi.org/10.1046/j.1365-2958.1996.5441070.x

45.Peeters, N., Carrère, S., Anisimova, M., Plener, L., Cazalé, A.-C., Genin, S., 2013. Repertoire, unified nomenclature and evolution of the Type III effector gene set in the Ralstonia solanacearum species complex. BMC Genomics 14, 859. https://doi.org/10.1186/1471-2164-14-859

46.Pensec, F., Lebeau, A., Daunay, M.C., Chiroleu, F., Guidot, A., Wicker, E., 2015. Towards the identification of Type III effectors associated with Ralstonia solanacearum virulence on tomato and eggplant. Phytopathology 105, 1529-1544. https://doi.org/10.1094/PHYTO-06-15-0140-R

47.Pérez-Losada, M., Crandall, K.A., Zenilman, J., Viscidi, R.P., 2007. Temporal trends in gonococcal population genetics in a high prevalence urban community. Infection, Genetics and Evolution 7, 271278. https://doi.org/10.1016/j.meegid.2006.11.003 
648

649

650

651

652

653

654

655

656

657

658

659

660

661

662

663

664

665

666

48.Perrier, A., Barlet, X., Peyraud, R., Rengel, D., Guidot, A., Genin, S., 2018. Comparative transcriptomic studies identify specific expression patterns of virulence factors under the control of the master regulator PhcA in the Ralstonia solanacearum species complex. Microbial Pathogenesis 116, 273-278. https://doi.org/10.1016/j.micpath.2018.01.028

49.Peyraud, R., Cottret, L., Marmiesse, L., Gouzy, J., Genin, S., 2016. A resource allocation trade-off between virulence and proliferation drives metabolic versatility in the plant pathogen Ralstonia solanacearum. PLOS Pathogens 12, e1005939. https://doi.org/10.1371/journal.ppat.1005939

50.Pierson, L.S., Pierson, E.A., 2010. Metabolism and function of phenazines in bacteria: impacts on the behavior of bacteria in the environment and biotechnological processes. Applied Microbiology and Biotechnology 86, 1659-1670. https://doi.org/10.1007/s00253-010-2509-3.

51.Ramos, I., Dietrich, L.E.P., Price-Whelan, A., Newman, D.K., 2010. Phenazines affect biofilm formation by Pseudomonas aeruginosa in similar ways at various scales. Research in Microbiology 161, 187-191. https://doi.org/10.1016/j.resmic.2010.01.003.

52.Roux, C., Pauwels, M., Ruggiero, M.-V., Charlesworth, D., Castric, V., Vekemans, X., 2013. Recent and ancient signature of balancing selection around the S-locus in Arabidopsis halleri and A. lyrata. Molecular Biology and Evolution 30, 435-447. https://doi.org/10.1093/molbev/mss246

53.Rozas, J., Ferrer-Mata, A., Sánchez-DelBarrio, J.C., Guirao-Rico, S., Librado, P., Ramos-Onsins, S.E., Sánchez-Gracia, A., 2017. DnaSP 6: DNA equence polymorphism nalysis of large data sets. Molecular Biology and Evolution 34, 3299-3302. https://doi.org/10.1093/molbev/msx248.

54.Safni, I., Cleenwerck, I., De Vos, P., Fegan, M., Sly, L., Kappler, U., 2014. Polyphasic taxonomic revision of the Ralstonia solanacearum species complex: proposal to emend the descriptions of Ralstonia solanacearum and Ralstonia syzygii and reclassify current $R$. syzygii strains as Ralstonia syzygii subsp. syzygii subsp. nov., $R$. solanacearum phylotype IV strains as Ralstonia syzygii subsp. indonesiensis subsp. nov., banana blood disease bacterium strains as Ralstonia syzygii subsp. celebesensis subsp. nov. and $R$. solanacearum phylotype I and III strains as Ralstonia pseudosolanacearum sp. nov. International Journal of Systematic and Evolutionary Microbiology 64, 3087-3103. https://doi.org/10.1099/ijs.0.066712-0.

55.Salanoubat, M., Genin, S., Artiguenave, F., Gouzy, J., Mangenot, S., Arlat, M., Billault, A., Brottier, P., Camus, J.C., Cattolico, L., Chandler, M., Choisne, N., Claudel-Renard, C., Cunnac, S., Demange, N., Gaspin, C., Lavie, M., Moisan, A., Robert, C., Saurin, W., Schiex, T., Siguier, P., Thébault, P., Whalen, M., Wincker, P., Levy, M., Weissenbach, J., Boucher, C.A., 2002. Genome sequence of the plant pathogen Ralstonia solanacearum. Nature 415, 497-502. https://doi.org/10.1038/415497a. 
680

56.Siewert, K.M., Voight, B.F., 2017. Detecting long-term balancing selection using allele frequency correlation. Molecular Biology and Evolution 34, 2996-3005.

$$
\text { https://doi.org/10.1093/molbev/msx209. }
$$

57.Siri, M.I., Sanabria, A., Boucher, C., Pianzzola, M.J., 2014. New type IV pili-related genes involved in early stages of Ralstonia solanacearum potato infection. Molecular Plant-Microbe Interactions 27, 712-724. https://doi.org/10.1094/MPMI-07-13-0210-R

58.Stoeckel, S., Klein, E.K., Oddou-Muratorio, S., Musch, B., Mariette, S., 2012. Microevolution of s-allele frequencies in wild cherry populations: respective impacts of negative frequency dependent selection and genetic drift: selection versus genetic drift at the s-locus between two generations. Evolution 66, 486-504. https://doi.org/10.1111/j.1558-5646.2011.01457.x.

59.Stukenbrock, E.H., McDonald, B.A., 2009. Population genetics of fungal and oomycete effectors involved in gene-for-gene interactions. Molecular Plant-Microbe Interactions 22, 371-380.

$$
\text { https://doi.org/10.1094/MPMI-22-4-0371 }
$$

60.Tajima, F. 1989. Statistical Method for Testing the Neutral Mutation Hypothesis by DNA

$$
\text { Polymorphism. Genetics 123, 585-95. }
$$

61.Tan, B.K., Bogdanov, M., Zhao, J., Dowhan, W., Raetz, C.R.H., Guan, Z., 2012. Discovery of a cardiolipin synthase utilizing phosphatidylethanolamine and phosphatidylglycerol as substrates. Proceedings of the National Academy of Sciences 109, 16504-16509. https://doi.org/10.1073/pnas.1212797109.

62.Tetteh, K.K.A., Stewart, L.B., Ochola, L.I., Amambua-Ngwa, A., Thomas, A.W., Marsh, K., Weedall, G.D., Conway, D.J., 2009. Prospective identification of malaria parasite genes under balancing selection. PLoS ONE 4, e5568. https://doi.org/10.1371/journal.pone.0005568.

63.The UniProt Consortium, 2017. UniProt: the universal protein knowledgebase. Nucleic Acids Research 45, D158-D169. https://doi.org/10.1093/nar/gkw1099.

64.Thomas, J.C., Godfrey, P.A., Feldgarden, M., Robinson, D.A., 2012. Candidate targets of balancing selection in the genome of Staphylococcus aureus. Molecular Biology and Evolution 29, 1175-1186. https://doi.org/10.1093/molbev/msr286.

65.Thompson, T.A., Brown, P.D., 2017. Association between the agr locus and the presence of virulence genes and pathogenesis in Staphylococcus aureus using a Caenorhabditis elegans model. International Journal of Infectious Diseases 54, 72-76. https://doi.org/10.1016/j.ijid.2016.11.411. 66.Torres, A.G., 2009. The cad locus of Enterobacteriaceae: More than just lysine decarboxylation. Anaerobe 15, 1-6. https://doi.org/10.1016/j.anaerobe.2008.05.002. 
67.Torres, A.G., Kaper, J.B., 2003. Multiple elements controlling adherence of enterohemorrhagic Escherichia coli 0157:H7 to HeLa cells. Infection and Immunity 71, 4985-4995. https://doi.org/10.1128/IAl.71.9.4985-4995.2003

68.Tran, M.T., MacIntyre, A., Khokhani, D., Hawes, M., Allen, C., 2016. Extracellular DNases of Ralstonia solanacearum modulate biofilms and facilitate bacterial wilt virulence. Environmental Microbiology 18, 4103-4117. https://doi.org/10.1111/1462-2920.13446.

69.Watterson, G.A., 1975. On the number of segregating sites in genetical models without recombination. Theoretical Population Biology 7, 256-276. https://doi.org/10.1016/00405809(75)90020-9

70.Yoshimochi, T., Hikichi, Y., Kiba, A., Ohnishi, K., 2009. The global virulence regulator PhcA negatively controls the Ralstonia solanacearum hrp regulatory cascade by repressing expression of the PrhIR signaling proteins. Journal of Bacteriology 191, 3424-3428. https://doi.org/10.1128/JB.01113-08

71.Zhang, L., Thomas, J.C., Didelot, X., Robinson, D.A., 2012. Molecular Signatures Identify a Candidate Target of Balancing Selection in an arcD-Like Gene of Staphylococcus epidermidis. Journal of Molecular Evolution 75, 43-54. https://doi.org/10.1007/s00239-012-9520-5

72.Zhou, Z., McCann, A., Weill, F.-X., Blin, C., Nair, S., Wain, J., Dougan, G., Achtman, M., 2014. Transient Darwinian selection in Salmonella enterica serovar paratyphi A during 450 years of global spread of enteric fever. Proceedings of the National Academy of Sciences 111, 12199-12204. https://doi.org/10.1073/pnas.1411012111. 
TABLES

732 Table 1. RSSC genomic sequences used in this analysis and population parameters and summary statistics calculated for whole replicons sequence data.

\begin{tabular}{|c|c|c|c|c|c|c|c|c|c|}
\hline $\begin{array}{l}\text { Phylotype/ } \\
\text { replicon }\end{array}$ & $\begin{array}{c}\text { Number of } \\
\text { genomes } \\
\text { analyzed }\end{array}$ & $\begin{array}{c}\text { Number of } \\
\text { nucleotides } \\
\text { analyzed }\end{array}$ & $\begin{array}{c}\text { Percentaje } \\
\text { of GMI1000 } \\
\text { replicon }^{a}\end{array}$ & $\begin{array}{c}\rho \\
\text { (per } \\
\text { site) }{ }^{b}\end{array}$ & $\begin{array}{c}\theta \\
\text { (per } \\
\text { site) }{ }^{b}\end{array}$ & $\rho / \theta$ & $\begin{array}{c}\text { Tajima's } \\
\text { D }\end{array}$ & $\begin{array}{c}\theta_{w} \\
\text { (per } \\
\text { site) }{ }^{c}\end{array}$ & $\begin{array}{c}\text { Fu-Li's } \\
\text { D* }\end{array}$ \\
\hline I/chromosome & 20 & 1907685 & 51.33 & 0.0120 & 0.0052 & 2.399 & -0.438 & 0.0051 & -0.676 \\
\hline I/megaplasmid & 20 & 1282321 & 61.22 & 0.0233 & 0.0067 & 3.470 & -0.450 & 0.0067 & -0.743 \\
\hline IIA/chromosome & 12 & 1971855 & 53.06 & 0.0008 & 0.0103 & 0.071 & 1.084 & 0.0101 & 0.895 \\
\hline IIA/megaplasmid & 7 & 938400 & 44.80 & 0.0020 & 0.0164 & 0.125 & -0.642 & 0.0160 & -0.725 \\
\hline IIB/chromosome & 20 & 1451109 & 39.05 & 0.0007 & 0.0092 & 0.071 & 0.923 & 0.0080 & 0.539 \\
\hline IIB/megaplasmid & 20 & 927177 & 44.27 & 0.0010 & 0.0133 & 0.075 & 0.930 & 0.0116 & 0.522 \\
\hline IV/chromosome & 5 & 1957952 & 52.68 & 0.0011 & 0.0112 & 0.088 & -0.434 & 0.0104 & -0.467 \\
\hline IV/megaplasmid & 5 & 503195 & 24.02 & 0.0021 & 0.0139 & 0.136 & -0.420 & 0.0134 & -0.468 \\
\hline
\end{tabular}

$734{ }^{a}$ As a reference, the GMI1000 chromosome has 3,716,413 bp and the megaplasmid 2,094,509 bp (ref. 55).

$735 b \rho$ and $\theta$ : per site recombination and mutation rate, respectively.

$736 c \theta_{\mathrm{w}}$ : Watterson's estimate of theta 
738 Table 2. Identity and probable function of genes showing highest observed values of three statistics $\left(\theta_{\mathrm{w}}\right.$, 739 Tajima's D, and Fu \& Li's D*) in the genome-wide analysis of RSSC phylotypes.

\begin{tabular}{|c|c|c|c|c|c|c|c|}
\hline \multirow[b]{2}{*}{$\begin{array}{l}\text { Phylotype/ } \\
\text { replicon }\end{array}$} & \multirow[b]{2}{*}{ Gene ID ${ }^{a}$} & \multirow[b]{2}{*}{$\begin{array}{l}\text { Gene } \\
\text { name }\end{array}$} & \multirow{2}{*}{$\begin{array}{l}\text { Number of } \\
\text { significant } \\
\text { hits }^{b}\end{array}$} & \multicolumn{3}{|c|}{ Summary statistics ${ }^{c}$} & \multirow[b]{2}{*}{ Gene description/function } \\
\hline & & & & $\theta_{w}$ & $\begin{array}{c}\text { Tajima's } \\
\text { D }\end{array}$ & $\begin{array}{c}\text { Fu \& Li's } \\
\text { D* }\end{array}$ & \\
\hline I/chromosome & RSc2735 & $p h c B$ & 5 & $0.0661 * *$ & $1.6873 * *$ & $1.7108^{* *}$ & $\begin{array}{l}\text { Class I SAM-dependent } \\
\text { methyltransferase }\end{array}$ \\
\hline I/chromosome & RSc2736 & phcs & 2 & $0.0729 * *$ & $1.6139 * *$ & $1.7266^{* *}$ & $\begin{array}{l}\text { Two-component sensor } \\
\text { histidine kinase }\end{array}$ \\
\hline I/chromosome & RSc0688 & - & 1 & $0.0482 * *$ & $2.9588^{* *}$ & $1.6747^{* *}$ & Glycosyl transferase \\
\hline I/chromosome & RSc2066 & - & 4 & $0.0595 * *$ & $3.4633^{* *}$ & $1.7026^{* *}$ & $\begin{array}{l}\text { Haloacid dehalogenase-like } \\
\text { hydrolase }\end{array}$ \\
\hline I/chromosome & RSc2670 & $\operatorname{Irg} B$ & 1 & $0.0154^{* *}$ & $2.2028 * *$ & $1.4372^{* *}$ & $\begin{array}{l}\text { Effector of murein hydrolase } \\
\text { transmembrane protein }\end{array}$ \\
\hline I/chromosome & RSc2669 & $\operatorname{Irg} A$ & 1 & $0.0210 * *$ & $2.4378^{* *}$ & $1.1771^{*}$ & Effector of murein hydrolase \\
\hline I/megaplasmid & RSp0832 & uxuL & 1 & $0.0155^{* *}$ & $1.6730^{* *}$ & $1.4372^{* *}$ & $\begin{array}{l}\text { Glucuronolactone/galactaro } \\
\text { lactone lactonase }\end{array}$ \\
\hline I/megaplasmid & RSp0304 & $\operatorname{rip} D$ & 2 & $0.0352 * *$ & $1.5729 * *$ & $1.4062 * *$ & $\begin{array}{l}\text { Type III effector protein, } \\
\text { avrPphD family }\end{array}$ \\
\hline I/megaplasmid & RSp0487 & ripA4 & 1 & $0.0183^{* *}$ & $2.5310^{* *}$ & $1.4823^{* *}$ & $\begin{array}{l}\text { Type III effector protein } \\
\text { (formerly AWR4) }\end{array}$ \\
\hline I/megaplasmid & RSp1212 & ripU & 2 & $0.1156^{* *}$ & $1.4848^{* *}$ & $1.4752^{* *}$ & $\begin{array}{l}\text { Type III effector protein } \\
\text { Probable pulA pullulanase }\end{array}$ \\
\hline I/megaplasmid & RSp0238 & $\operatorname{glg} X$ & 1 & $0.0296 * *$ & $2.3832 * *$ & $1.3369 * *$ & $\begin{array}{l}\text { related glycosidase protein, } \\
\text { glycogen debranching } \\
\text { enzyme }\end{array}$ \\
\hline I/megaplasmid & RSp1530 & - & 1 & $0.0944 * *$ & $1.5829 * *$ & $1.6620^{* *}$ & $\begin{array}{l}\text { Polyphenol oxidase (laccase) } \\
\text { oxidoreductase }\end{array}$ \\
\hline I/megaplasmid & RSp1100 & - & 1 & $0.0493 * *$ & $2.1856^{* *}$ & $1.3555^{* *}$ & $\begin{array}{l}\text { Putative signal sensing } \\
\text { transmembrane protein, } \\
\text { phosphorelay sensor kinase } \\
\text { activity }\end{array}$ \\
\hline IIA/chromosome & RCFBP_11371 & paaH2 & 1 & $0.0298 * *$ & $2.0233^{* *}$ & $1.5364^{* *}$ & $\begin{array}{l}\text { Putative 3-hydroxybutyryl- } \\
\text { coA dehydrogenase } \\
\text { oxidoreductase }\end{array}$ \\
\hline IIA/chromosome & RCFBP_11349 & - & 1 & $0.0364 * *$ & $2.1164 * *$ & $1.5632^{* *}$ & $\begin{array}{l}\text { Putative high-affinity } \\
\text { branched-chain amino acid } \\
\text { transport system permease } \\
\text { (liv operon) }\end{array}$ \\
\hline IIA/chromosome & RCFBP_20503 & parc & 1 & $0.0381 * *$ & $1.8461^{* *}$ & $1.5686^{* *}$ & $\begin{array}{l}\text { DNA topoisomerase IV, } \\
\text { subunit A }\end{array}$ \\
\hline IIA/chromosome & RCFBP_11056 & $a d i$ & 1 & $0.0248 * *$ & $2.1501 * *$ & $1.5085^{* *}$ & Lysine decarboxylase \\
\hline IIA/chromosome & RCFBP_10967 & iles & 1 & $0.0248 * *$ & $2.0846^{* *}$ & $1.5085^{* *}$ & Isoleucine--tRNA ligase \\
\hline IIA/chromosome & RCFBP_21311 & $\arg C$ & 1 & $0.0232 * *$ & $1.9107^{* *}$ & $1.4970 * *$ & $\begin{array}{l}\mathrm{N} \text {-acetyl-gamma-glutamyl- } \\
\text { phosphate reductase }\end{array}$ \\
\hline IIA/chromosome & RCFBP_10305 & & 1 & $0.0282 * *$ & $1.9675^{* *}$ & $1.5280 * *$ & $\begin{array}{l}\text { Putative transcription } \\
\text { regulator protein }\end{array}$ \\
\hline IIA/chromosome & RCFBP_10218 & soxF & 1 & $0.0248 * *$ & $2.0192 * *$ & $1.5085^{* *}$ & $\begin{array}{l}\text { Sulfide dehydrogenase } \\
\text { [flavocytochrome c] } \\
\text { flavoprotein chain precursor }\end{array}$ \\
\hline IIA/chromosome & RCFBP_11858 & bioA & 1 & $0.0265^{* *}$ & $1.9424 * *$ & $1.5188^{* *}$ & $\begin{array}{l}\text { Adenosylmethionine--8- } \\
\text { amino-7-oxononanoate }\end{array}$ \\
\hline
\end{tabular}




\begin{tabular}{|c|c|c|c|c|c|c|c|}
\hline IIA/chromosome & RCFBP_10092 & - & 1 & $0.0381 * *$ & $1.9520 * *$ & $1.5686 * *$ & $\begin{array}{l}A B C \text { transmembrane type- } 1 \\
\text { domain }\end{array}$ \\
\hline IIA/chromosome & RCFBP_10712 & phcQ & 1 & $0.0282 * *$ & $1.9908 * *$ & $1.5280 * *$ & Response regulator receiver \\
\hline IIA/chromosome & RCFBP_10711 & - & 2 & $0.0450 * *$ & $2.0770 * *$ & $1.5866 * *$ & Putative methyltransferase \\
\hline IIA/chromosome & RCFBP_21242 & - & 1 & $0.0414 * *$ & $2.5492 * *$ & $1.5782 * *$ & $\begin{array}{l}\text { Putative isomerase, with } \\
\mathrm{PhzC} / \mathrm{PhzF} \text { domain }\end{array}$ \\
\hline IIA/chromosome & RCFBP_20936 & srkA & 1 & $0.0298 * *$ & $2.0897 * *$ & $1.5364 * *$ & Stress response kinase $A$ \\
\hline IIA/chromosome & RCFBP_10686 & ripW & 2 & $0.0911 * *$ & $2.0865^{* *}$ & $1.5603 * *$ & Type III effector protein \\
\hline IIA/chromosome & RCFBP_11806 & $\operatorname{rip} G 4$ & 2 & $0.0381 * *$ & $2.0578 * *$ & $1.5686 * *$ & Type III effector protein \\
\hline IIA/chromosome & RCFBP_11870 & $\operatorname{rip} M$ & 1 & $0.0298 * *$ & $2.1673 * *$ & $1.5364 * *$ & Type III effector protein \\
\hline IIA/chromosome & RCFBP_20594 & ripS5 & 1 & $0.0265 * *$ & $1.9424 * *$ & $1.5188 * *$ & Type III effector protein \\
\hline IIA/megaplasmid & $\operatorname{RCFBP}_{7} \mathrm{mp1031}$ & суaB & 1 & $0.0939 * *$ & 0.9326 & $1.5961^{* *}$ & $\begin{array}{l}\text { ABC transporter (cyclolysin- } \\
\text { type) }\end{array}$ \\
\hline IIA/megaplasmid & RCFBP_mp1060 & - & 1 & $0.0776 * *$ & $1.4690^{*}$ & 1.1125 & Putative adhesin/hemolysin \\
\hline IIA/megaplasmid & RCFBP_mp3003 & cls & 1 & $0.2490 * *$ & $1.7362 * *$ & $1.6971 * *$ & Cardiolipin synthase A \\
\hline IIA/megaplasmid & $\begin{array}{c}\text { RCFBP_mp3011 } \\
9\end{array}$ & - & 1 & $0.0653 * *$ & $1.2030 *$ & $1.4386 * *$ & $\begin{array}{l}\text { Putative type IV fimbrial } \\
\text { biogenesis protein pilY1 with } \\
\text { A-like domain }\end{array}$ \\
\hline IIA/megaplasmid & $\frac{\text { RCFBP_mp3043 }}{8}$ & ripF1 & 1 & $0.1 * *$ & 1.0458 & $1.5313^{* *}$ & $\begin{array}{l}\text { Type III effector protein } \\
\text { (formerly PopF1) }\end{array}$ \\
\hline IIA/megaplasmid & $\frac{\text { RCFBP_mp2000 }}{3}$ & - & 1 & $0.0551 * *$ & $1.6353 * *$ & 1.0059 & $\begin{array}{l}\text { Bacteriophage-related } \\
\text { protein of unknown function }\end{array}$ \\
\hline IIB/chromosome & RSPO_c00124 & atpH & 1 & $0.0226 * *$ & $2.1129 * *$ & $1.5336 * *$ & $\begin{array}{l}\text { ATP synthase, f1 sector } \\
\text { subunit delta }\end{array}$ \\
\hline IIB/chromosome & RSPO_c00113 & livK & 1 & $0.0211 * *$ & $2.0978 * *$ & $1.5182 * *$ & $\begin{array}{l}\text { Leucine-specific binding } \\
\text { precursor transmembrane } \\
\text { protein }\end{array}$ \\
\hline IIB/chromosome & RSPO_c00179 & $g c l$ & 1 & $0.0240 * *$ & $2.1552^{* *}$ & $1.5475^{* *}$ & $\begin{array}{l}\text { Tartronate-semialdehyde } \\
\text { synthase (glyoxylate } \\
\text { carboligase) }\end{array}$ \\
\hline IIB/chromosome & $\begin{array}{l}\text { RSPO_c00415 } \\
\text { RSPO_c00416 }\end{array}$ & - & 1 & $0.0352 * *$ & $2.0846 * *$ & $1.4063^{* *}$ & $\begin{array}{l}\text { b-ketoadipate enol-lactone } \\
\text { hydrolase protein and 3- } \\
\text { ketoacyl-(acyl-carrier- } \\
\text { protein) reductase }\end{array}$ \\
\hline IIB/chromosome & RSPO_c00497 & $\sec Y$ & 1 & $0.0282 * *$ & $1.9898 * *$ & $1.5826 * *$ & $\begin{array}{l}\text { Preprotein translocase } \\
\text { (membrane subunit) }\end{array}$ \\
\hline IIB/chromosome & RSPO_c00765 & $p h c B$ & 1 & $0.0240 * *$ & $2.1264 * *$ & $1.5475^{* *}$ & Regulatory protein \\
\hline IIB/chromosome & RSPO_c02646 & pilX & 1 & $0.0211 * *$ & $2.0335^{* *}$ & $1.5182 * *$ & $\begin{array}{l}\text { Putative type IV pili } \\
\text { assembly protein }\end{array}$ \\
\hline IIB/chromosome & RSPO_c01209 & - & 1 & $0.0282 * *$ & $2.7986 * *$ & $1.5826^{* *}$ & $\begin{array}{l}\text { 1-deoxy-d-xylulose-5- } \\
\text { phosphate synthase protein }\end{array}$ \\
\hline IIB/chromosome & RSPO_c01332 & $\operatorname{ripAJ}$ & 2 & $0.0226 * *$ & $2.2473 * *$ & $1.5336 * *$ & Type III effector protein \\
\hline IIB/chromosome & RSPO_c02391 & $\| d P$ & 1 & $0.0183 * *$ & $1.9736 * *$ & $1.4823 * *$ & I-lactate permease protein \\
\hline IIB/chromosome & RSPO_c02306 & lepA & 1 & $0.0268 * *$ & $2.0272 * *$ & $1.5718 * *$ & $\begin{array}{l}\text { GTP-binding elongation } \\
\text { factor }\end{array}$ \\
\hline IIB/chromosome & RSPO_c01998 & $\operatorname{rip} G 7$ & 3 & $0.0804 * *$ & $2.0276 * *$ & $1.4278^{* *}$ & $\begin{array}{l}\text { Type III effector protein } \\
\text { (formerly GALA7) }\end{array}$ \\
\hline
\end{tabular}




\begin{tabular}{|c|c|c|c|c|c|c|c|}
\hline IIB/chromosome & RSPO_c01999 & ripG6 & 1 & $0.0268 * *$ & $2.6851^{* *}$ & $1.5718^{* *}$ & $\begin{array}{l}\text { Type III effector protein } \\
\text { (formerly GALA6) }\end{array}$ \\
\hline IIB/chromosome & RSPO_c01798 & $\operatorname{aid} B$ & 1 & $0.0536 * *$ & $2.2941 * *$ & $1.3902 * *$ & $\begin{array}{l}\text { Isovaleryl CoA } \\
\text { dehydrogenase }\end{array}$ \\
\hline IIB/chromosome & RSPO_c01795 & $\operatorname{fad} B$ & 1 & $0,0338 * *$ & $2.0162 * *$ & $1.6180 * *$ & $\begin{array}{l}\text { Fused 3-hydroxybutyryl-CoA } \\
\text { epimerase }\end{array}$ \\
\hline \multirow{2}{*}{$\begin{array}{l}\text { IIB/chromosome } \\
\text { IIB/chromosome }\end{array}$} & RSPO_c00909 & - & 1 & $0.0620 * *$ & $3.0207 * *$ & $1.5774 * *$ & Lipoprotein \\
\hline & RSPO_c01066 & metG1 & 1 & $0.0254^{* *}$ & $2.2792 * *$ & $1.5602 * *$ & Methionyl-tRNA synthetase \\
\hline IIB/chromosome & RSPO_c01082 & adi & 1 & $0.0338 * *$ & $2.5566 * *$ & $1.3908 * *$ & $\begin{array}{l}\text { Biodegradative arginine } \\
\text { decarboxylase protein }\end{array}$ \\
\hline IIB/chromosome & RSPO_c03170 & - & 1 & $0.0354 * *$ & $2.0256 * *$ & $1.5602 * *$ & Chromate transport protein \\
\hline IIB/chromosome & RSPO_c03029 & - & 1 & $0.0312 * *$ & $1.9276 * *$ & $1.3562 * *$ & $\begin{array}{l}\text { Sensory box/GGDEF family } \\
\text { protein }\end{array}$ \\
\hline IIB/megaplasmid & RSPO_m01227 & fabl & 1 & $0.0466 * *$ & $2.2681^{* *}$ & $1.6703^{* *}$ & $\begin{array}{l}\text { Enoyl-[acyl-carrier-protein] } \\
\text { reductase (NADH) }\end{array}$ \\
\hline IIB/megaplasmid & $\begin{array}{l}\text { RSPO_m01150 } \\
\text { RSPO_m01151 }\end{array}$ & pehc & 1 & $0.0409 * *$ & $2.3366^{* *}$ & $1.4587^{* *}$ & $\begin{array}{l}\text { Gluconolactonase and } \\
\text { polygalacturonase proteins }\end{array}$ \\
\hline IIB/megaplasmid & RSPO_m00202 & ripH2 & 9 & $0.0776 * *$ & $2.2152^{* *}$ & $1.7322 * *$ & Type III effector protein \\
\hline IIB/megaplasmid & RSPO_m00035 & ripG3 & 1 & $0.0676 * *$ & $2.3026^{* *}$ & $1.4768^{* *}$ & $\begin{array}{l}\text { Type III effector protein } \\
\text { (formerly GALA3) }\end{array}$ \\
\hline IIB/megaplasmid & RSPO_m01206 & ripAO & 1 & $0.0494 * *$ & $2.1286 * *$ & $1.6788 * *$ & Type III effector protein \\
\hline IIB/megaplasmid & RSPO_m01229 & ripS3 & 3 & $0.0409 * *$ & $1.9967^{* *}$ & $1.6505^{* *}$ & $\begin{array}{l}\text { Type III effector protein } \\
\text { (formerly SKWP3) }\end{array}$ \\
\hline IIB/megaplasmid & RSPO_m01312 & ripZ & 1 & $0.0747^{* *}$ & $2.9580 * *$ & $1.7286^{* *}$ & Type III effector protein \\
\hline IIB/megaplasmid & RSPO_m01371 & $\operatorname{ripC1}$ & 1 & $0.0338 * *$ & $2.5836 * *$ & $1.3908 * *$ & Type III effector protein \\
\hline IIB/megaplasmid & RSPO_m00869 & $\operatorname{rip} N$ & 1 & $0.0620 * *$ & $2.0883^{* *}$ & $1.5774 * *$ & Type III effector protein \\
\hline \multirow{2}{*}{$\begin{array}{l}\text { IIB/megaplasmid } \\
\text { IIB/megaplasmid }\end{array}$} & RSPO_m00770 & $\operatorname{rip} A R$ & 2 & $0.0380 * *$ & $2.2529 * *$ & $1.6388^{* *}$ & Type III effector protein \\
\hline & RSPO_m01600 & $\operatorname{ripBH}$ & 3 & $0.0620 * *$ & $2.7880 * *$ & $1.7082 * *$ & Type III effector protein \\
\hline IIB/megaplasmid & RSPO_m01541 & ripF1 & 2 & $0.0366 * *$ & $2.1843^{* *}$ & $1.6322 * *$ & $\begin{array}{l}\text { Type III effector protein } \\
\text { (formerly PopF1) }\end{array}$ \\
\hline IV/chromosome & RPSI07_1784 & - & 2 & $0.0312 * *$ & $1.5828^{* *}$ & $1.5828^{* *}$ & 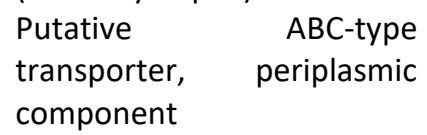 \\
\hline IV/chromosome & RPSI07_2871 & tyrs & 2 & $0.0312 * *$ & $1.5828 * *$ & $1.5828 * *$ & Tyrosyl-tRNA synthetase \\
\hline IV/chromosome & RPSI07_1208 & $r p o D$ & 3 & $0.0768^{* *}$ & $1.8719 * *$ & $1.8719 * *$ & $\begin{array}{l}\text { RNA polymerase sigma70 } \\
\text { factor }\end{array}$ \\
\hline IV/chromosome & RPSI07_1185 & galU & 2 & $0.048^{* *}$ & $1.6941 * *$ & $1.6941 * *$ & $\begin{array}{l}\text { Glucose-1-phosphate } \\
\text { uridylyltransferase }\end{array}$ \\
\hline IV/chromosome & RPSI07_0660 & $\operatorname{mraY}$ & 1 & $0.384 * *$ & $1.6419 * *$ & $1,6419 * *$ & $\begin{array}{l}\text { Phospho-N- } \\
\text { acetylmuramoyl- } \\
\text { pentapeptide transferase }\end{array}$ \\
\hline IV/chromosome & RPSI07_0072 & ripE1_1 & 2 & $0,1056 * *$ & $1.6690 * *$ & $1.6690 * *$ & Type III effector protein \\
\hline IV/chromosome & RPSI07_0735 & ripW & 3 & $0.1056 * *$ & $1.9186 * *$ & $1.6690 * *$ & Type III effector protein \\
\hline IV/megaplasmid & RPSI07_mp0105 & - & 1 & $0.1464^{* *}$ & $1.7880 * *$ & $1.7880^{* *}$ & Putative acetyltransferase \\
\hline IV/megaplasmid & RPSI07_mp0022 & $c / c B$ & 1 & $0.0624 * *$ & $1.6238^{* *}$ & $1.6238^{* *}$ & $\begin{array}{l}\text { Chloride channel clcB-like } \\
\text { protein }\end{array}$ \\
\hline $\begin{array}{l}\text { respectivel } \\
{ }_{b} \text { Number } 0 \\
{ }^{c} \text { Observed } \\
\text { and }{ }^{* *} p<\end{array}$ & $\begin{array}{l}\text { Ignificant windows } \\
\text { lues of statistics for }\end{array}$ & each gene & SI & of coalesce & simulation & ing standar & hylotype I, IIA, IIB or IV \\
\hline 745 & & & & & & & \\
\hline
\end{tabular}


bioRxiv preprint doi: https://doi org/10.1101/388207- this version posted August 9 2018. The copyright holder for this preprint (which was not certified by peer review) is the author/funder, who has granted bioRxiv a license to display the preprint in perpetuity. It is made available under aCC-BY-NC-ND 4.0 International license.

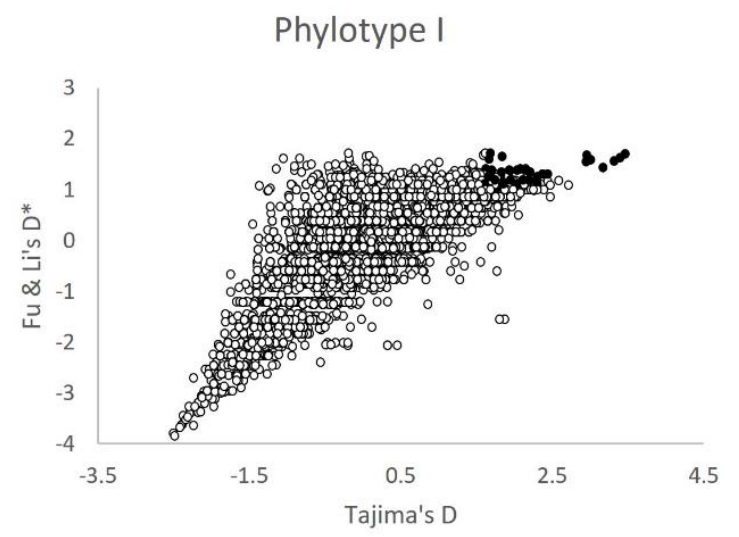

Phylotype IIB

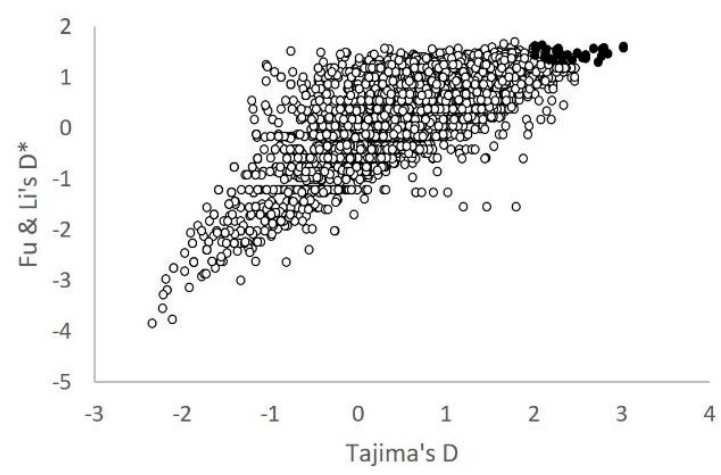

Phylotype IIA

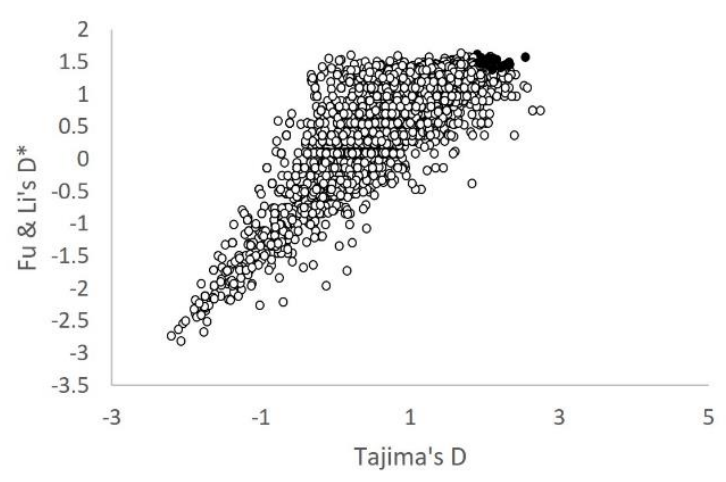

Phylotype IV

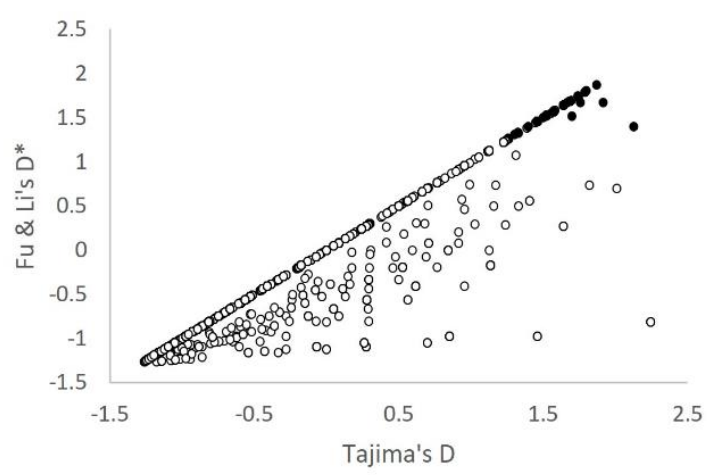

747

748 Figure 1. Two-dimensional plot of Tajima's D and Fu \& Li's D* values for all windows. Shaded dots represent 749 the $5 \%$ top windows of the distribution of both measures of BS. 


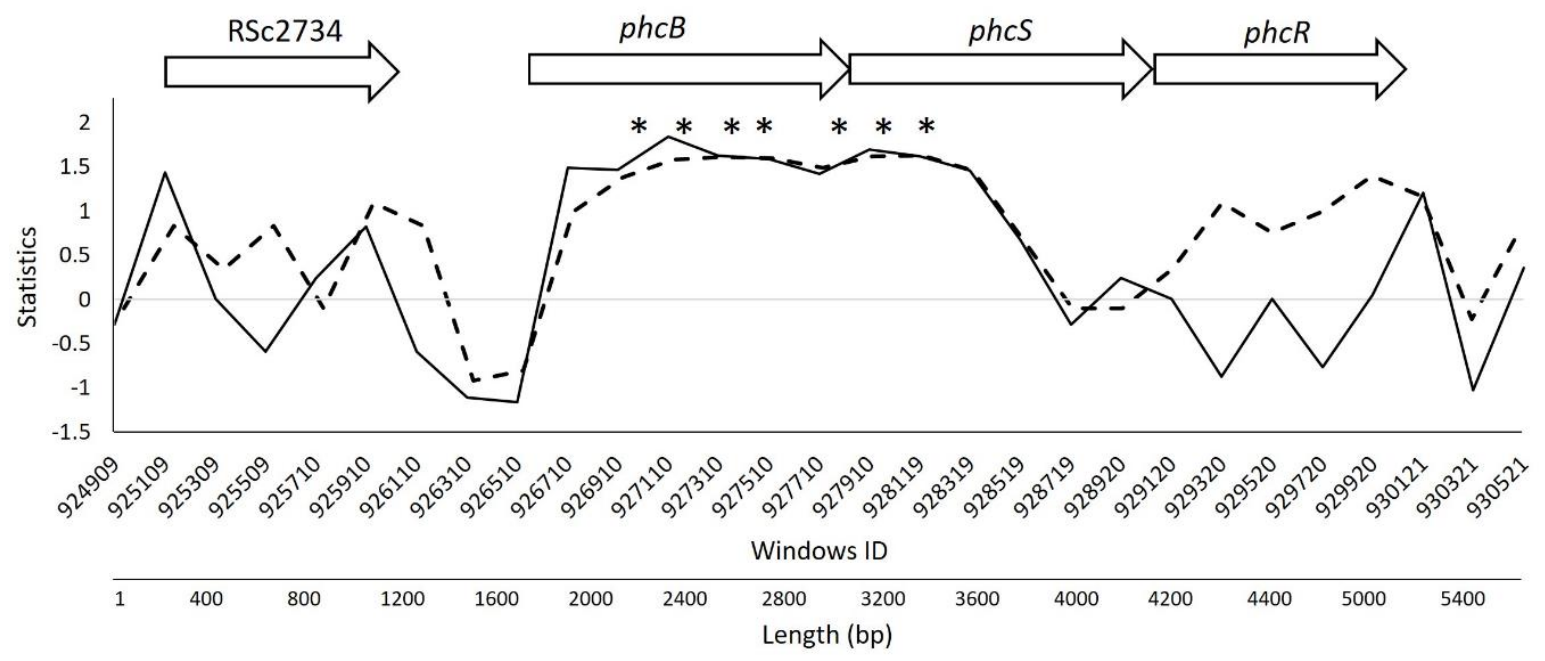

752 Figure 2. Analysis of genomic region corresponding to the phcBSR operon in strain GMI1000 showing sliding window analyses for two statistics: Tajima's D (solid line) and Fu \& Li's D* (dotted line). All three genes of the operon comprise about $3.9 \mathrm{~Kb}$ of the genome; however, the gene (RSc2734) physically preceding this operon is also shown for comparison purposes. Windows are $200 \mathrm{bp}$ and asterisks indicate the windows with extreme values of respective statistics. Arrows represent gene arrangement in the genome of strain GMI1000. 